\title{
Detailed Electrode Catheter Positioning is Important for the Ablation of Outflow Tract Origin Ventricular Arrhythmias
}

In Geol Song, MD1; Sung-Hwan Kim, MD2; Ju Youn Kim, MD3; Jeong Ho Kim, MD4; Yoo Ri Kim, MD5; TaeSeok Kim, MD6; Ji-Hoon Kim, MD7; Sung-Won Jang, MD, $\mathrm{PhD}^{8}$; Man Young Lee, MD, PhD9; Tai-Ho Rho, $\mathrm{MD}, \mathrm{PhD}^{8}$; Yong-Seog Oh, MD, PhD²

${ }^{1}$ Division of Cardiology, Heart center, Konyang University Hospital, Daejeon, Korea

${ }^{2}$ Division of Cardiology, Department of Internal Medicine, College of Medicine, The Catholic University of Korea, Seoul St. Mary's Hospital

${ }^{3}$ Division of Cardiology, Department of Internal Medicine, College of Medicine, The Catholic University of Korea, Uijeongbu St. Mary's Hospital

${ }^{4}$ Department of Cardiology, Pohang St. Mary's Hospital ${ }^{5}$ Division of Cardiology, Department of Internal Medicine, College of Medicine, The Catholic University of Korea, Incheon St. Mary's Hospital ${ }^{6}$ Division of Cardiology, Department of Internal Medicine, College of Medicine, The Catholic University of Korea, Daejeon St. Mary's Hospital ${ }^{7}$ Division of Cardiology, Department of Internal Medicine, College of Medicine, The Catholic University of Korea, St. Vincent's Hospital ${ }^{8}$ Division of Cardiology, Department of Internal Medicine, College of Medicine, The Catholic University of Korea, St. Paul's Hospital

${ }^{9}$ Division of Cardiology, Department of Internal Medicine, College of Medicine, The Catholic University of Korea, Yeouido St. Mary's Hospital

Received: November 11, 2017

Revision Received: November 21, 2017

Accepted: December 8, 2017

Correspondence: Yong-Seog Oh, MD, PhD

Division of Cardiology, Department of Internal

Medicine, College of Medicine, Seoul St. Mary's

Hospital, The Catholic University of Korea,

Banpo-daero 222, Seocho-gu, Seoul 06591,

Republic of Korea

Tel: +82-2-2258-1141 FAX: +82-2-591-1506

E-mail: oys@catholic.ac.kr

Copyright (C) 2017 The Official Journal of Korean Heart Rhythm Society Editorial Board and EUM \& Communications

\begin{abstract}
Background and Objectives: Electroanatomical mapping using a three-dimensional (3D) system has high accuracy and improves the results of the ablation of outflow tract (OT) premature ventricular contraction (PVC) or ventricular tachycardia (VT) but imposes a considerable economic burden. Here, we compared detailed diagnostic catheterization and 3D mapping system for the ablation of OT PVC/VT.
\end{abstract}

Materials and Methods: Between June 2012 and February 2017, patients with symptomatic OT PVC/VT underwent radiofrequency ablation. Group 1 underwent detailed diagnostic catheterization (using circular and linear multielectrodes) without a 3D mapping system, while group 2 underwent diagnostic catheterization using a conventional 3D mapping system. Procedural success of PVC reduction, remaining symptoms, need for post-operative medications, and procedural time were evaluated.

Results: Ninety-eight OT PVC/VT cases were consecutively enrolled. The mean follow-up period was $17.7 \pm 14.5$ months. Neither acute success rate $(95 \%$ vs. $82 \%, p=0.06)$ nor a PVC reduction $>$ $80 \%(84 \%$ vs. $87 \%, p=0.74)$ differed significantly between the two groups. The recurrence rates of PVC-related symptoms were similar $(12 \%$ vs. $7 \%, p=0.06)$ between the groups, but the medication requirement for symptomatic PVC differed (12\% vs. 29\%, $p<0.01$ ). The total procedure time of group 1 was shorter than that of group 2 $(132 \pm 42 \mathrm{~min}$ vs. $157 \pm 47 \mathrm{~min}, p=0.01)$ and fluoroscopy time $(24 \pm 15$ $\min$ vs. $38 \pm 22 \min , p<0.01)$ and ablation time $(528 \pm 538 \mathrm{sec}$ vs. $899 \pm 598 \mathrm{sec}, p<0.01)$ were also significantly shortened.

Conclusion: Detailed electrode catheter positioning is a safe and cost-effective method for the ablation of OT PVCNT.

Key Words: - Outflow Tract - Premature Ventricular Contraction - Catheter Ablation 


\section{Introduction}

Ventricular outflow tract (OT)-originating premature ventricular contraction (PVC) constitutes a considerable proportion of clinically encountered PVC and causes various symptoms of dizziness, palpitations, and chest discomfort. OT PVC and right ventricular outflow tract (RVOT) ventricular tachycardia (VT) account for $10 \%$ of cases of idiopathic $\mathrm{VT}^{1}$ and do not commonly cause tachycardia-induced cardiomyopathy.

To control this kind of PVC or VT, catheter ablation is known to be an effective treatment modality. ${ }^{2-4}$ However, localization of the origin of OT PVC/VT relevant to procedural success is not always guaranteed in the ventricular OT of a three-dimensional (3D) narrow tubular structure. To enhance mapping accuracy, ablation guided by a 3D system or a body surface electrocardiographic mapping system as a noninvasive technique is quite safe and effective for the ablation of OT PVC/VT., ${ }^{5,6}$ However, these modalities carry considerable economic burden. The efficacy of multielectrode catheter mapping methods has been reported in some studies but these methods have the limitations of small group sizes and uncertain statistical significance. $^{7,8}$

Our study sought to evaluate the efficacy of circular and multielectrode diagnostic catheters without 3D system mapping (detailed catheter positioning group) for the ablation of OT PVC/VT compared to that of the conventional 3D system mapping method.

\section{Materials and Methods}

\section{Study population}

This retrospective non-randomized study compared different mapping methods used for the ablation of OT PVC/VT. Between June 2012 and February 2017, a total of 92 patients who were admitted for an electrophysiology (EP) study and ablation due to OT PVC/VT were consecutively enrolled in this study. All patients had documented 12-lead electrocardiography (ECG) of the OT PVC/VT with symptoms including dizziness, palpitation, dyspnea, or chest discomfort and had problems using antiarrhythmic medications due to drug inefficacy or intolerance. Patients underwent 24-hour Holter monitoring before the EP study and again 3-6 months after the procedure.

\section{Mapping and ablation procedure}

The EP study was performed in a fasting and non-sedated state. All antiarrhythmic medications were stopped for five half-lives before the procedure. The EP study and ablation were performed using two different mapping methods. In the group of patients who underwent detailed diagnostic catheterization without 3D system mapping (Group 1), linear multielectrode catheters (Livewire; St. Jude Medical, St. Paul, MN, USA) and circular multielectrode catheter (Inquiry Optima; St. Jude Medical) with ventricular OT angiogram) were used. In the other group (Group 2), 3D system mapping (CARTO, Biosense Webster or NavX; St. Jude Medical) was used. The detailed diagnostic catheter position (Group 1) is shown in Figure 1. The diagnostic procedure was performed as follows. After right ventricular OT angiography, the earliest activation point in the duodecapolar catheter was identified (vertical mapping in the OT). A circular catheter was positioned there to locate the earliest point (transverse mapping in the OT). The circular catheter was positioned through a prepositioned guiding introducer (Fast Cath; St. Jude Medical). The ablation was performed at the earliest activation point via the ablation electrode (Figure 1). Radiofrequency energies were delivered for 60-120 seconds with a power of $25-40 \mathrm{~W}$ at the site of origin. If the radiofrequency energy was delivered in an aortic cusp or within the coronary venous system, an initial power of 15-20 W was used. Acute success was defined as the absence of PVC at the end of the procedure.

\section{Follow-up}

After the procedure, the patients visited the outpatient department and were examined using a Holter monitor or serial ECG. Symptoms of PVC were evaluated together. Chronic success was defined as $>80 \%$ PVC reduction in follow-up Holter monitoring compared to the baseline study or more than two serial PVC-negative ECG at least 1 week apart. ${ }^{9,10}$ Primary outcomes were acute and chronic success. Secondary outcomes 

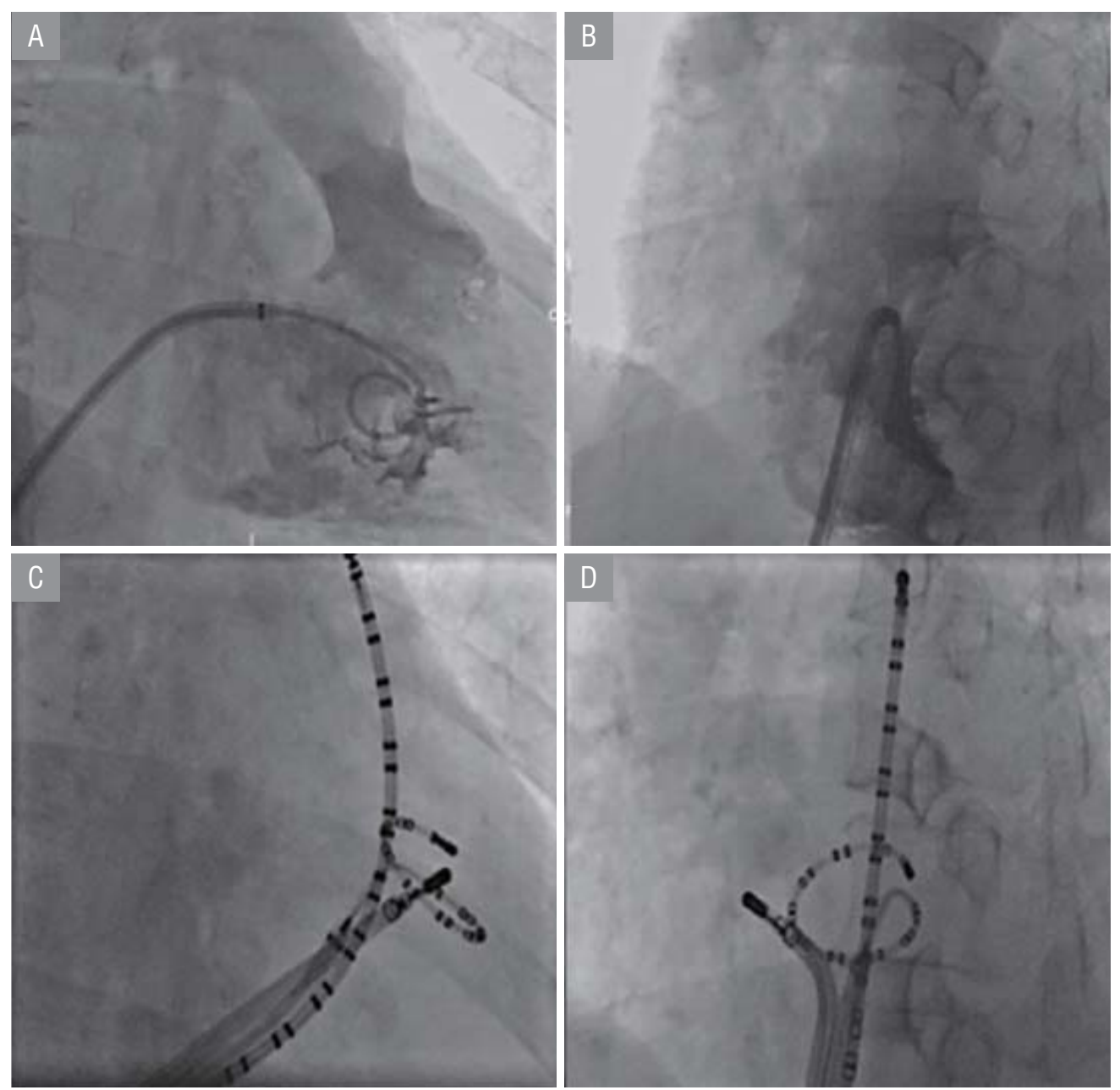

Figure 1. Detailed catheter position with duodeca and circular catheters with right ventricular outflow tract angiogram; (A) RAO view, (B) LAO view. Catheter position; (C) RAO view, (D) LAO view

were need for medication for symptom relief, duration of total procedure time, fluoroscopy time, ablation time, and presence of procedure-related complications.

\section{Statistical analysis}

Continuous variables are expressed as mean \pm standard deviation and were compared using Student's $t$-test. Categorical variables were compared using the $\chi^{2}$ test or Fisher's exact test or Cox regression analysis. The survival result was compared using Kaplan-Meier analysis. Statistical significance was considered when the $\mathrm{P}$ value was $<0.05$. The statistical analysis was performed using SPSS statistical software (SPSS version 18; SPSS, Chicago, IL, USA).

\section{Results}

A total of 92 de novo OT PVC/VT cases and six repeat cases were consecutively enrolled. The mean follow-up period was 17.7 \pm 14.5 months. The baseline demographic and clinical characteristics of the study population are shown in Table 1. Patients were younger in group 1, but the follow-up period was similar between the two groups. Group 1 had a shorter total procedure time, fluoroscopy time, and ablation time than group 2. Acute success rates were similar between the two groups ( $95 \%$ in group 1 vs. $82 \%$ in group $2, p=0.06)$. Chronic success rates $(84 \%$ in group 1 vs. $87 \%$ in group $2, p=0.74$ ) and recurrent symptoms ( $12 \%$ in group 1 vs. $7 \%$ in group $2, p=0.06)$ were similar. However, the medication requirement including beta blockers, 
Table 1. Comparison of baseline characteristics between detailed catheter positioned group and 3D mapping system groups

\begin{tabular}{lccc} 
& $\begin{array}{c}\text { Detailed catheter group } \\
(\mathrm{n}=38)\end{array}$ & $\begin{array}{c}3 \text { mapping group } \\
(\mathrm{n}=60)\end{array}$ & P-value \\
\hline Age (years) & $50.1 \pm 13.8$ & $56.7 \pm 16.2$ & 0.04 \\
Male & $7(18 \%)$ & $27(45 \%)$ & 0.09 \\
Follow up duration (months) & $16.3 \pm 15.2$ & $18.6 \pm 14.2$ & 0.44 \\
Premature complex classification & & & 0.01 \\
PVC & $25(66 \%)$ & $22(37 \%)$ & \\
Non-sustained VT & $13(34 \%)$ & $38(63 \%)$ & 0.03 \\
Origin & & & \\
RV-outtlow tract origin & $36(94 \%)$ & $45(75 \%)$ & \\
LV-outflow tract origin & $1(3 \%)$ & $13(22 \%)$ & \\
PVC morphology $\geq 2$ & $1(3 \%)$ & $2(3 \%)$ & 1.0 \\
Redo case & 2 & 4 & \\
\hline
\end{tabular}

Values are presented as $n(\%)$ and as mean $\pm S D$.

'P-value was obtained by Fisher's exact test.

$\mathrm{RV}$, right ventricle; LV, left ventricle; PVC, premature ventricular complex; VT, ventricular tachycardia.

non-dihydropyridine calcium channel blockers, and antiarrhythmic drugs was lower in group 1 (12\% in group 1 vs. $29 \%$ in group $2, p<0.01$ ) (Table 2). Cox regression analysis showed that the use of 3D mapping did not significantly affect procedural success (Table 3). Long-term arrhythmia-free survival did not differ significantly between the two groups (Figure 3). In group 2, two patients had VT that originated from the left ventricle (LV) summit; ablation was attempted in the target area but was insufficient.

Six patients underwent a repeat ablation procedure due to PVC recurrence (two in group 1, four in group 2). Among the three patients in group 1 , one underwent a repeated ablation procedure using the same initial detailed catheter mapping system, whereas the other patient opted for a 3D mapping system. Similarly, in group 2, one patient used the detailed catheter mapping system and the other three patients used a same initial 3D mapping system. Four patients were free from OT PVC/VT and other ventricular arrhythmias, but two patients experienced recurrence during the remaining follow-up period.

The total procedure time of group 1 was shorter than that of group 2 (132 $\pm 42 \mathrm{~min}$ vs. $157 \pm 47 \mathrm{~min}, p=0.01)$ and fluoroscopy time ( $24 \pm 15 \mathrm{~min}$ vs. $38 \pm 22 \mathrm{~min}, p<0.01)$ and ablation time $(528 \pm 538 \mathrm{sec}$ vs. $899 \pm 598 \mathrm{sec}, p<0.01)$ were also significantly shortened. The procedure-related cost of group 1 was also lower than that of group $2\left(3,432.7 \pm 132.9 \times 10^{3}\right.$ won vs. $16,883.8 \pm 11,530.2 \times 10^{3}$ won, $\left.p=0.1\right)$.

There were no major complications in this study group. In group 1, one patient developed a puncture site hematoma after the procedure that was managed conservatively with eventual selfresolution.

\section{Discussion}

Methods for mapping of the origin site of OT PVC/VT have been suggested in many studies. ${ }^{5.8,1-15}$ Our study results showed that mapping using the multielectrode catheter (including linear and circular) mapping inside RVOT is quite effective for the ablation of OT PVC/VT.

The multipolar linear electrode was placed crossing the pulmonic valve in the RVOT and circular multielectrode catheters could be manipulated along the cranio-caudal axis of the RVOT, allowing acquisition of the OT to identify the earliest 
Table 2. Difference of procedural outcomes between two groups

\begin{tabular}{lccc} 
& $\begin{array}{c}\text { Detailed catheter group } \\
(\mathrm{n}=38)\end{array}$ & $\begin{array}{c}3 \text { mapping group } \\
(\mathrm{n}=60)\end{array}$ & $P$-value \\
\hline Acute success & $36(95 \%)$ & $49(82 \%)$ & 0.06 \\
FU success & $32(84 \%)$ & $52(87 \%)$ & 0.74 \\
$\quad$ PVC reduction (>80 \%) & $21347 \pm 13310$ & $28061 \pm 54574$ & 0.52 \\
PVC number (pre) & $5273 \pm 8983$ & $4982 \pm 6740$ & 0.50 \\
PVC number (post) & $5(12 \%)$ & $18(7 \%)$ & 0.06 \\
Symptom remain & $5(12 \%)$ & $31(29 \%)$ & $<0.01$ \\
Medication requirement & $9(26 \%)$ & $24(31 \%)$ & 0.1 \\
Recurrence & $132 \pm 42$ & $157 \pm 47$ & 0.01 \\
Total procedure time (min) & $24 \pm 15$ & $38 \pm 22$ & $<0.01$ \\
Fluoroscopy time (min) & $528 \pm 538$ & $899 \pm 598$ & $<0.01$ \\
Ablation time (sec) & &
\end{tabular}

Values are presented as $n(\%)$ and as mean \pm SD.

'Recurrence means at least one of low PVC reduction, symptom, or anti-arrhythmic medication need

FU, follow up; PVC, premature ventricular complex; min, minute; sec, second

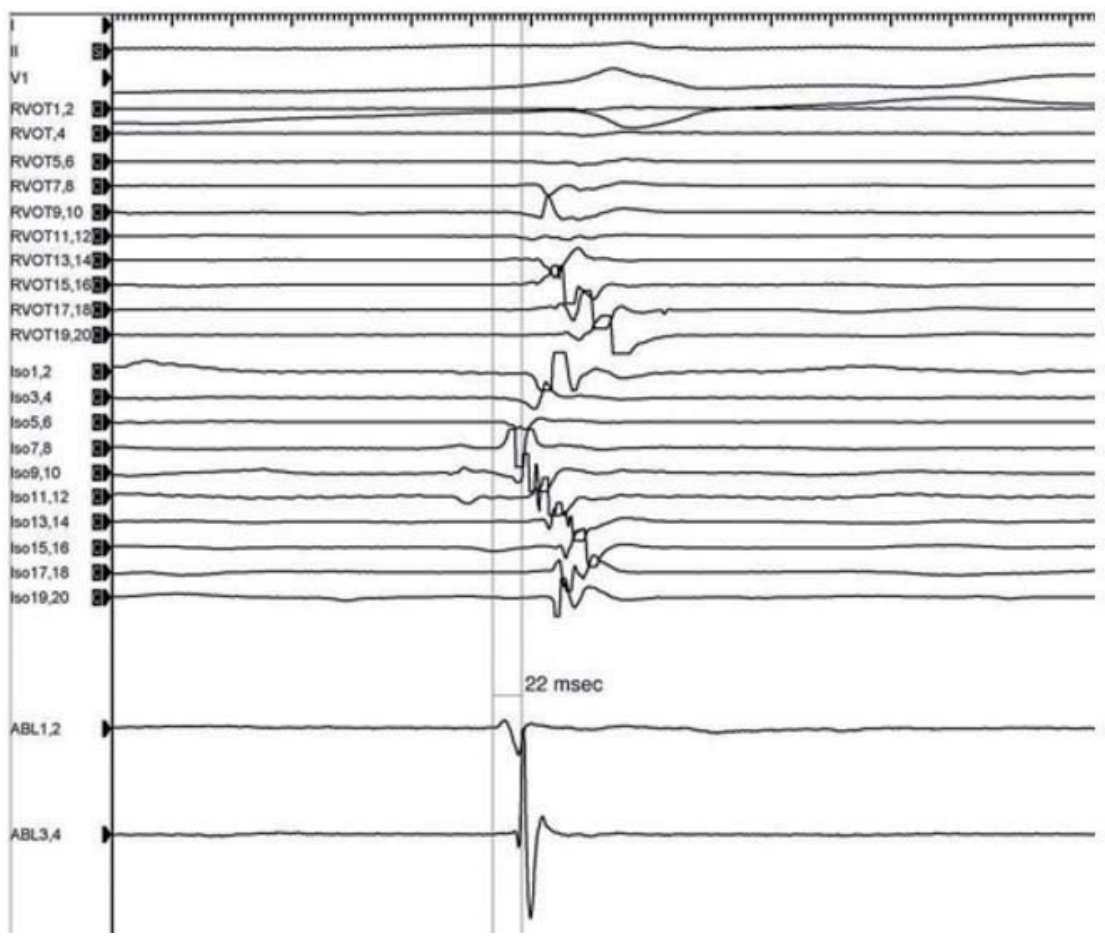

Figure 2. Electrogram showing the earliest potential by ablation catheter during premature ventricular contraction. That was prior to the earliest potential seen by the lasso catheter ( $22 \mathrm{msec}$ ).

RVOT, right ventricular outflow tract ; TDW, tip deflecting wire 
Table 3. Cox regression analysis of long term success

\begin{tabular}{lccc}
\hline & Hazard ratio & 95\% confidence interval & $P$-value \\
\hline Female & 0.44 & $0.13-1.52$ & 0.19 \\
Age & 0.95 & $0.90-1.02$ & 0.14 \\
Age $\geq 60$ & 2.81 & $0.33-24.0$ & 0.34 \\
Non-sustained VT & 0.95 & $0.22-4.08$ & 0.95 \\
3D mapping use & 0.29 & $0.05-1.6$ & 0.15 \\
LVOT origin & 0.18 & $0.02-2.01$ & 0.16 \\
\hline
\end{tabular}

LVOT, left ventricular outflow tract

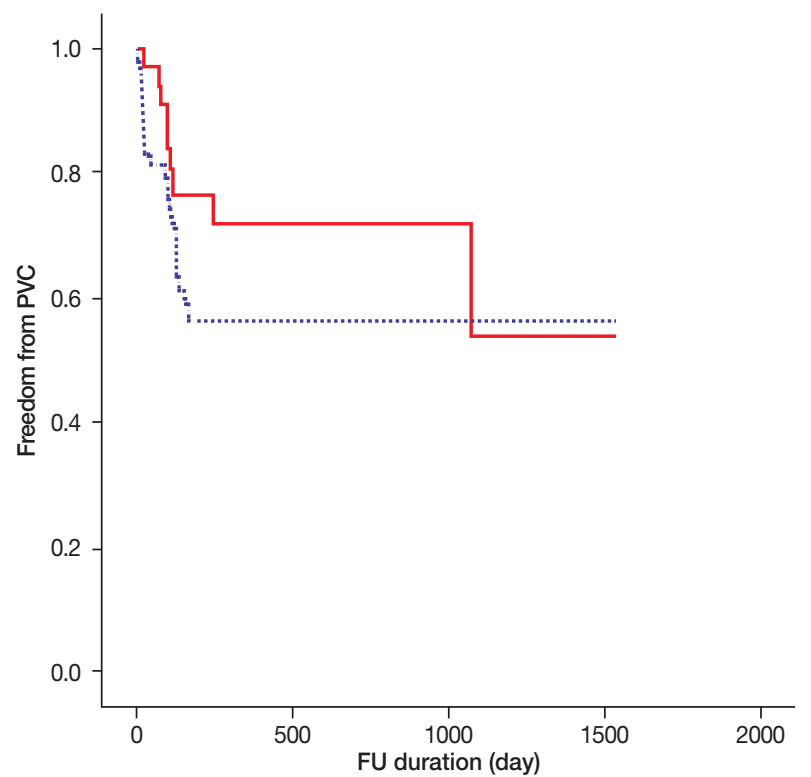

Figure 3. Kaplan-Meier survival curves for survival free from event. Red solid line indicate detailed diagnostic catheterization without three dimensional system mapping group and blue dot line indicate three dimensional system mapping group ( $p=0.22$; log rank test).

activation focus. The ablation catheter was introduced to determine and define a more precise origin site target.

Current 3D mapping systems have been used for catheter ablation of OT PVC/VT. They could allow repeated repositioning of the catheter to the optimal ablation site, resulting in more accurate ablation. Meanwhile, the 3D system mapping method has the limitation of high cost since it requires expensive equipment. On the contrary, our detailed mapping method has the advantage of cost effectiveness since it requires only an additional catheter and a guiding introducer.
In our detailed mapping practice, our main concern is the potential risk of circular multielectrode catheter entrapment in cardiac valves or subvalvular structures. Circular multielectrode catheter entrapments have been reported, especially in the ablation of atrial fibrillation. ${ }^{16}$ As described previously in another study, we used pre-shaped guiding introducers for the delivery and placement of circular multielectrode catheters to avoid catheter entrapment ${ }^{8}$; in this study, we did not note this complication.

Total procedure time, fluoroscopic time, and ablation time were significantly shorter in the detailed catheter position group than in the 3D mapping group, meaning that detailed catheter position mapping is a safe method. However, it may be not a simple comparison since more mapping and ablation time was needed for complex arrhythmia in 3D mapping group. In fact, there were more instances of non-sustained VT- and LVOT-origin ventricular arrhythmia in the 3D mapping group.

Previous studies showed an $85-100 \%$ acute success rate of OT PVC/VT ablation. ${ }^{12,17}$ The acute success rate of this study was similar and even slightly more favorable in the detailed catheter position group than the 3D mapping group, although the difference was not statistically significant. The detailed catheter positioning made more precise mapping possible for determining the ablation site in the RVOT.

The long-term PVC arrhythmia-free survival results did not different between the two groups. Neither PVC recurrence, a subjective symptom of PVC, nor the need for medication due to symptomatic PVC differed between groups. In this study, the proportion of patients who complained of recurrent symptoms 
seemed to be relatively high, but it might have been overestimated because we could not confirm that their recurrent symptoms were truly related with the recurrence of targeted PVC.

This study has some limitations in that the number of patients with non-sustained VT- and LV-outflow tract origin PVC/VT were significantly higher in the $3 \mathrm{D}$ mapping group than in the detailed catheter group. We thought that a non-sustained VT is more complex arrhythmia than PVC, while LVOT-origin PVC/ VT is also more difficult to ablate than RV-outflow origin PVC/ VT, so 3D mapping methods may be used more frequently in non-sustained VT or LV-outflow origin ventricular arrhythmia ablation procedures.

\section{Conclusions}

Detailed electrode catheter positioning is a safe and costeffective method for the ablation of OT PVC/VT.

\section{Conflict of interest}

The authors declare no conflicts of interest. Informed consent was obtained from all individual study participants.

\section{Acknowledgements}

The authors thank to contributors (Jeong Wook Park and Young Woong $\mathrm{Ha}$ ) for their technical assistance of electrophysiologic study and radiofrequency ablation procedure in cardiac catheterization laboratory.

\section{References}

1) Aliot EM, Stevenson WG, Almendral-Garrote JM, Bogun F, Calkins CH, Delacretaz E, Bella PD, Hindricks G, Jaïs P, Josephson ME, Kautzner J, Kay GN, Kuck KH, Lerman BB, Marchlinski F, Reddy V, Schalij MJ, Schilling R, Soejima K, Wilber D; European Heart Rhythm Association; European Society of Cardiology; Heart Rhythm Society. EHRA/HRS expert consensus on catheter ablation of ventricular arrhythmias: developed in a partnership with the European Heart Rhythm Association (EHRA), a registered branch of the European Society of Cardiology (ESC), and the Heart Rhythm Society (HRS); in collaboration with the American
College of Cardiology (ACC) and the American Heart Association (AHA). Europace. 2009;11:771-817.

2) Grimm W, Menz V, Hoffmann J, Maisch B. Reversal of tachycardia induced cardiomyopathy following ablation of repetitive monomorphic right ventricular outflow tract tachycardia. Pacing Clin Electrophysiol. 2001;24:166-171.

3) Mountantonakis SE, Frankel DS, Gerstenfeld EP, Dixit S, Lin D, Hutchinson MD, Riley M, Bala R, Cooper J, Callans D, Garcia F, Zado ES, Marchlinski FE. Reversal of outflow tract ventricular premature depolarization-induced cardiomyopathy with ablation: effect of residual arrhythmia burden and preexisting cardiomyopathy on outcome. Heart Rhythm. 2011;8:1608-1614.

4) Nakagawa M, Takahashi N, Nobe S, Ichinose M, Ooie T, Yufu F, Shigematsu S, Hara M, Yonemochi H, Saikawa T. Gender differences in various types of idiopathic ventricular tachycardia. $J$ Cardiovasc Electrophysiol. 2002;13:633-638.

5) Friedman PA, Asirvatham SJ, Grice S, Glikson M, Munger TM, Rea RF, Shen WK, Jahanghir A, Packer DL, Hammill SC. Noncontact mapping to guide ablation of right ventricular outflow tract tachycardia. J Am Coll Cardiol. 2002;39:1808-1812.

6) Fung J, Chan H, Chan J, Chan W, Kum L, Sanderson J. Ablation of nonsustained or hemodynamically unstable ventricular arrhythmia originating from the right ventricular outflow tract guided by noncontact mapping. Pacing Clin Electrophysiology. 2003;26:1699-1705.

7) Aiba T, Shimizu W, Taguchi A, Suyama K, Kurita T, Aihara N, Kamakura S. Clinical usefulness of a multielectrode basket catheter for idiopathic ventricular tachycardia originating from right ventricular outflow tract. J Cardiovasc Electrophysiol. 2001;12:511517.

8) Saleem MA, Burkett S, Passman R, Dibs S, Engelstein ED, Kadish $\mathrm{AH}$, Goldberger JJ. New simplified technique for 3d mapping and ablation of right ventricular outflow tract tachycardia. Pacing Clin Electrophysiol. 2005;28:397-403.

9) Baser K, Bas HD, Belardi D, Yokokawa M, Good E, Latchamsetty R, Morady F, Bogun F. Predictors of outcome after catheter ablation of premature ventricular complexes. J Cardiovasc Electrophysiol. 2014;25:597-601.

10) Baman TS, Lange DC, Ilg KJ, Gupta SK, Liu TY, Alguire C, Armstrong W, Good E, Chugh A, Jongnarangsin K, Pelosi F Jr, Crawford T, Ebinger M, Oral H, Morady F, Bogun F. Relationship between burden of premature ventricular complexes and left 
ventricular function. Heart Rhythm. 2010;7:865-869.

11) Calkins. H, Kalbfleisch. SJ, El-Atassi. R, Langberg. JJ, Morady F. Relation between efficacy of radiogfrequency catheter ablation and site of origin of idiopathic ventricular tachycardia. Am J Cardiol. 1993;71:827-833.

12) Ribbing M, Wasmer K, Mönnig G, Kirchhof P, Loh P, Breithardt G, Haverkamp W, Eckardt L. Endocardial mapping of right ventricular outflow tract tachycardia using noncontact activation mapping. J Cardiovasc Electrophysiol. 2003;14:602-608.

13) Erkapic D, Neumann T. Ablation of premature ventricular complexes exclusively guided by three-dimensional noninvasive mapping. Card Electrophysiol Clin. 2015;7:109-115.

14) Erkapic D, Greiss H, Pajitnev D, Zaltsberg S, Deubner N, Berkowitsch A, Möllman S, Sperzel J, Rolf A, Schmitt J, Hamm CW, Kuniss M, Neumann T. Clinical impact of a novel threedimensional electrocardiographic imaging for non-invasive mapping of ventricular arrhythmias-a prospective randomized trial. Europace. 2015;17:591-597.

15) Jamil-Copley S, Bokan R, Kojodjojo P, Qureshi N, Koa-Wing M, Hayat S, Kyriacou A, Sandler B, Sohaib A, Wright I, Davies DW, Whinnett Z, S Peters N, Kanagaratnam P, Lim PB. Noninvasive electrocardiographic mapping to guide ablation of outflow tract ventricular arrhythmias. Heart Rhythm. 2014;11:587-594.

16) Kesek M, Englund A, Jensen SM, Jensen-Urstad M. Entrapment of circular mapping catheter in the mitral valve. Heart Rhythm. 2007;4:17-19.

17) Coggins DL, Lee RJ, Sweeney J, Chein WW, Van Hare G, Epstein L, Gonzalez R, Griffin JC, Lesh MD, Scheinman MM. Radiofrequency catheter ablation as a cure for idiopathic tachycardia of both left and right ventricular origin. J Am Coll Cardiol. 1994;23:1333-1341. 